\title{
EVENTOS
}

\section{COLOQUIO DISTRITAL DE MATEMÁTICAS Y ESTADÍSTICA}

El Coloquio Distrital de Matemáticas y Estadística, que llega este año a su versión $\mathrm{XVI}$, es un evento anual organizado por las universidades Nacional de Colombia. Pedagógica Nacional y Distrital Francisco José de Caldas, con el ánimo de reunir a las comunidades de matemáticos, estadísticos y docentes en las diferentes actividades que se desarrollan dentro del certamen

Son objetivos del evento:

- Contribuir carla actualización y profundización de los conocimientos de todas aquellas personas cuyo quehacer guarde estrecha relación con la matemática o su enseñanza

- Brindar a oportunidad de divulgar los trabajos de investigación que se han realizado e intercambiar los resultados en los campos investigativo, docente $\mathrm{o}$ de aplicaciones

- Facilitar un espacio académico en donde todas las personas inquietas por la matemática o su enseñanza puedan discutir sobre sus temas de interés

Este año el evento se realizará del 29 de noviembre al 3 de diciembre en las instalaciones de la Universidad Nacional, en donde se ofrecerán 30 cursillos en tres diferentes horarios para que cada asistente tenga la oportunidad de seleccionar 3 de acuerdo con sus inquietudes y preferencias. De cada uno de ellos es posible conseguir la publicación correspondiente.

Se ofrece además una amplia gama de conferencias en diferentes tópicos de la matemática y su enseñanza, así como foros y seminarios en temas de interés general.

Su participación en la elaboración de propuestas de cursillos, talleres, comunicaciones o conferencias que sean de interés para docentes de cualquier nivel educativo, investigadores o personas que de una u otra manera se sirvan de la matemática o la estadística, puede tramitarse por intermedio de alguno de los Departamentos de Matemática de las Universidades organizadoras. Igualmente las inscripciones para participar como asistente y las informaciones que se requieran sobre el evento pueden ser solicitadas en ellos.

Universidad Pedagógica Nacional Departamento de Matemáticas. Oficina B-306. Tel: 3473562/67/70. Ext: 254

Universidad Nacional de Colombia Departamento de Matemáticas y Estadística. Tel: 3165000. Ext: 13162

Universidad Distrital

"Francisco José de Caldas" Departamento de Matemáticas.

Tel: 2842373 